\title{
Cognitive Inhibition and Cognitive Load: A Moderation Hypothesis
}

\author{
Tony Yeigh \\ Southern Cross University, New South Wales, Australia
}

\begin{abstract}
Cognitive Load Theory (CLT) has seen widespread acceptance within education as an approach to instructional design that supports the broader theory of a limited capacity working memory (WM) system. However a problem exists in relation to CLT, in that it has not been able to offer a causal mechanism that clearly explains how the theory works. This report discusses a pilot study investigating cognitive inhibition as the mechanism by which extrinsic cognitive load may be explained. The results of the study showed that the ability to inhibit distracting information was distributed across a normal range of individual differences for a group of year-8 ( $8^{\text {th }}$ grade $)$ students, and that these differences influenced how cognitive load was experienced by the students in relation to their learning. These findings suggest that inhibition moderates at least the extrinsic form of cognitive load, and offer further direction in terms of the empirical testing of CLT.
\end{abstract}

\section{Introduction}

Cognitive Load Theory (CLT) is a theory of instructional design based upon assumptions associated with what is commonly referred to as the working memory (WM) system. From an information processing perspective, CLT uses WM to explain how to design for effective learning in terms of the limited processing capacity of WM. Thus CLT represents a model of instructional design, wherein the processing constraints of WM are used to explain differences in student learning within an information processing framework.

A well-established relationship exists between $\mathrm{WM}$ and classroom practice, stemming from a body of research that connects both literacy-based and numeracy-based domains of learning to the way WM is used to store and process instructional information within the constraints of individual differences. Because of this, individual differences in WM form an important aspect of instructional design, where the instruction must seek to account for the limited processing capacity of the WM system as efficiently as possible.

A fundamental tenet of CLT is that the limited processing capacity of WM shapes the way learning takes place. This occurs because the architecture of information processing, in particular the relationship between WM and long-term memory (LTM), largely determines learning efficiency [1;2]. In line with this, the goal of CLT is to provide instructional design principles that facilitate learning in accordance with the limited capacity architecture of the WM system. Schnotz and Kürschner [12] list the basic assumptions of CLT as including:

- a multiple memory store architecture,

- the notion that long-term memory consists of cognitive schemata,

- the construct of "cognitive load" (intrinsic, extraneous, and germane) as mental effort, due to the allocation of limited WM resources,

- the notion of 'learning' as an increase in expertise, due to schematic alterations in LTM,

- the concept of 'understanding' as the coherent, simultaneous processing of information elements at the WM level,

- the idea of "instructional consequences" - that instruction acts as an executive guide to assist in the development of relevant schemata for the learner.

It is important to note that in relation to cognitive load, each distinct load type (intrinsic, extraneous, and germane) contributes to mental effort in a different manner. Intrinsic load refers to the complexity of learning information, and is produced by the number of discrete elements that interact within WM during a learning task. Extraneous load stems from the format of instructional encoding (the organisation and delivery of instructional information), and is viewed as a possible impediment to learning that can occur due to poor design and organisation of the learning information. Germane 
load (also referred to as essential load) refers to the amount of mental effort devoted to learning taskrelevant information. This type of cognitive load is considered appropriate and desirable, as it consolidates memory construction. All three load types are important to instructional design, because they all connect the limited resources of WM to LTM via schema acquisition and/or schematic automaticity [13].

A problem exists with the current construct of CLT however, wherein some theorists suggest the theory lacks an explanatory mechanism that is necessary and sufficient to the scope of its theoretical application $[6 ; 12]$. From this perspective, the present article reports on a pilot study which sought to investigate whether the neuro-cognitive process known as cognitive inhibition can provide a necessary causal mechanism for CLT, on the basis that inhibition appears to explain the impact of cognitive load upon limited capacity processing. It is also postulated that inhibition can provide a sufficient causal mechanism for CLT, because it is able to explain how this impact occurs in relation to the processing of instructional information.

\subsection{Defining inhibition}

Cognitive inhibition is a term widely used by cognitive and neuro-cognitive psychologists to refer to the ability to suppress distracting or non-relevant information during on-task cognitive engagement [7]. The term inhibition is used here to refer to the inhibitory function generally associated with WM, and is operationally defined as a functional connection between items of information where, when two or more items are competing for attentional focus, one of the items needs to be suppressed [9].

The goal of the pilot study was to establish whether or not inhibition exerts a distinguishable influence on cognitive load. This is considered a necessary first step in reconceptualising the relationship between Cognitive Load Theory (CLT) and existing knowledge concerning information processing. In addition, because CLT represents a design-based approach to the implementation of WM, such a reconceptualisation may also have implications for instructional design. Due to such considerations, it is necessary to examine the relationship between inhibition and cognitive load in some detail.

\section{The role of instructional priming}

In information processing terms, the amount of mental effort an individual experiences in relation to an on-task activity is affected by differences in the levels of activation and inhibition involved in a learning task $[8 ; 13]$. In this respect extraneous cognitive load is especially pertinent to Cognitive Load Theory (CLT), because it conjoins the influence of instructional encoding to the experience of mental effort. As implicit instructional designers, teachers often encode information in a particular way for student learning, yet this may not always represent the most effective processing approach in terms of cognitive load.

Such instructional issues involve the degree to which instruction makes particular load-type demands upon WM, which, according to Mayer and Moreno, derive from instructional priming. In this context priming refers to the way information is cognitively activated for ongoing processing, with a bias for positive priming (increased efficiency in the way the information is accessed), or for negative priming (decreased efficiency in the way the information is accessed), occurring due to the way antecedent information is used to organise for ontask processing. An important consideration for cognitive load is, therefore, how the teacher encodes instructional information to prime for more-or-less efficient activation of the information to be learned. Of importance to the current study is that excessive extraneous cognitive load may be expected whenever the teacher's encoding of instructional information involves too much negative priming. This is because negative priming requires the learner to perform significant non-essential processing in the form of incidental (task-irrelevant) processing and representational (short-term memory) holding. Figure 1 provides a conceptual representation of this set of relationships. Note that priming appears to control the relationship between extraneous and germane cognitive load. 


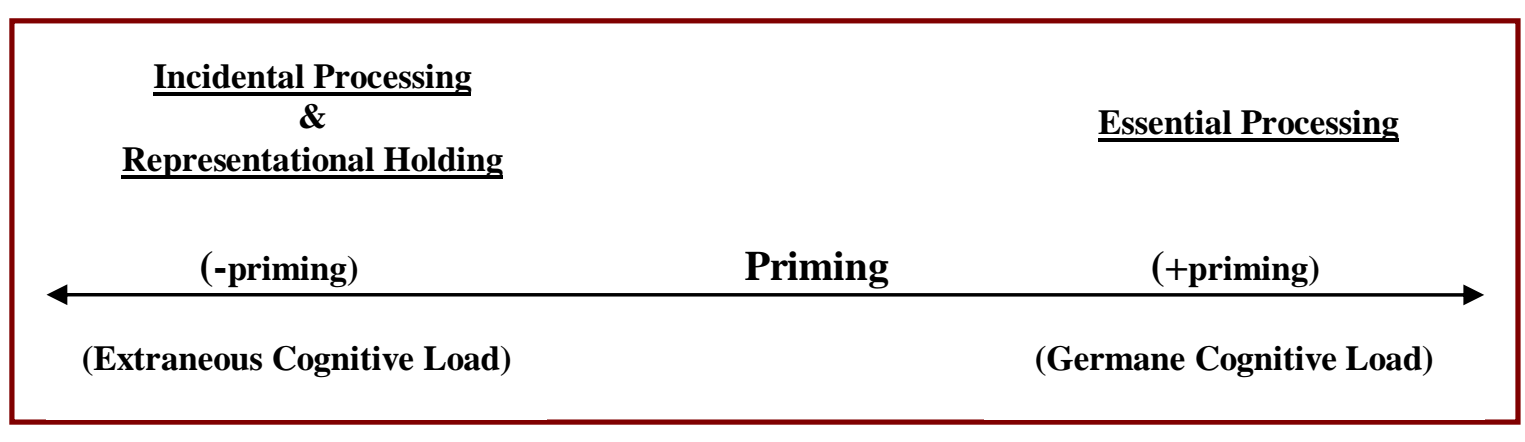

Figure 1: Conceptual representation of the relationship between cognitive load and priming

Importantly, non-essential processing requires increased inhibitory function because of the greater need to stop task-irrelevant and short-term information from interfering with the objectives of the learning task. In this understanding of instructional encoding, mental effort represents the primary measure of cognitive load because the instructional priming can be used to locate specific inhibition requirements along a processing continuum, from non-essential processing (extraneous cognitive load) to essential processing (germane cognitive load). Inhibition is thus positioned in relation to CLT because it highlights how differences in priming translate into differences in extraneous cognitive load. This position is supported by some cognitive load theorists [15], who have suggested that an important part of what cognitive load measures is the level of inhibitory function required by a particular task.

In line with this, the current study sought to determine whether the relationship between cognitive load and inhibition appears consistent, that is, whether measures of inhibitory function for individual students maintain a clear and predictable correspondence with the amount of cognitive load experienced by the students.

\section{Study rationale}

Most WM research has tended to highlight ways in which problematic task-relevant processing creates a susceptibility to poor performance on a specific task. In contrast, relatively little research has looked at ways in which the ability to suppress irrelevant information might also contribute to poor learning at the more generalised level of classroom learning, and in relation to specific measures of inhibition. The value of the current study rests in the fact that it seeks to identify whether inhibition does interact with the amount of mental effort experienced by individual students in relation to their learning in a systematic fashion. The study thus seeks to determine whether inhibition constitutes a causal mechanism for extrinsic cognitive load.

\section{The investigation}

This research addressed the relationship between inhibitory differences and cognitive load for a group of year- 8 ( $8^{\text {th }}$ grade $)$ students. WM functions relative to representational holding (short-term store), and problem-solving ability (accuracy in solving math problems) were determined for the students, as these functions relate to the extrinsic and germane cognitive load types. Using a Flanker task, the ability to process conflicting or distracting information was also determined for the students, and self-reported measures of cognitive load were obtained in relation to instruction the students received from four different teachers, all of whom taught the students in common. Of particular interest was whether those students less able to inhibit distracting information would rate the instruction as requiring higher levels of mental effort than those students more able to inhibit distracting information, as well as whether these ratings would vary or remain stable across different teachers. The main, underlying question being addressed was, "Do individual differences in inhibitory ability affect the way students experience mental effort at the classroom level?".

Figure 2 provides a conceptual overview of how the relationships between WM, inhibition, and cognitive load are understood to interact within the study. Note that inhibition (CI) is viewed as an aspect of WM, and as such interacts with the instructional encoding received from different teachers to moderate the amount of cognitive load (CL) students experience in relation to the teachers. Thus, it is proposed that individual differences in inhibitory ability act to moderate mental effort in relation to received instruction, to produce a range of differences in the cognitive load experienced by learners. It is therefore expected that clear correspondences will occur between measured 
inhibition for the students (using the Flanker task) and the cognitive load ratings they assign to the teachers.

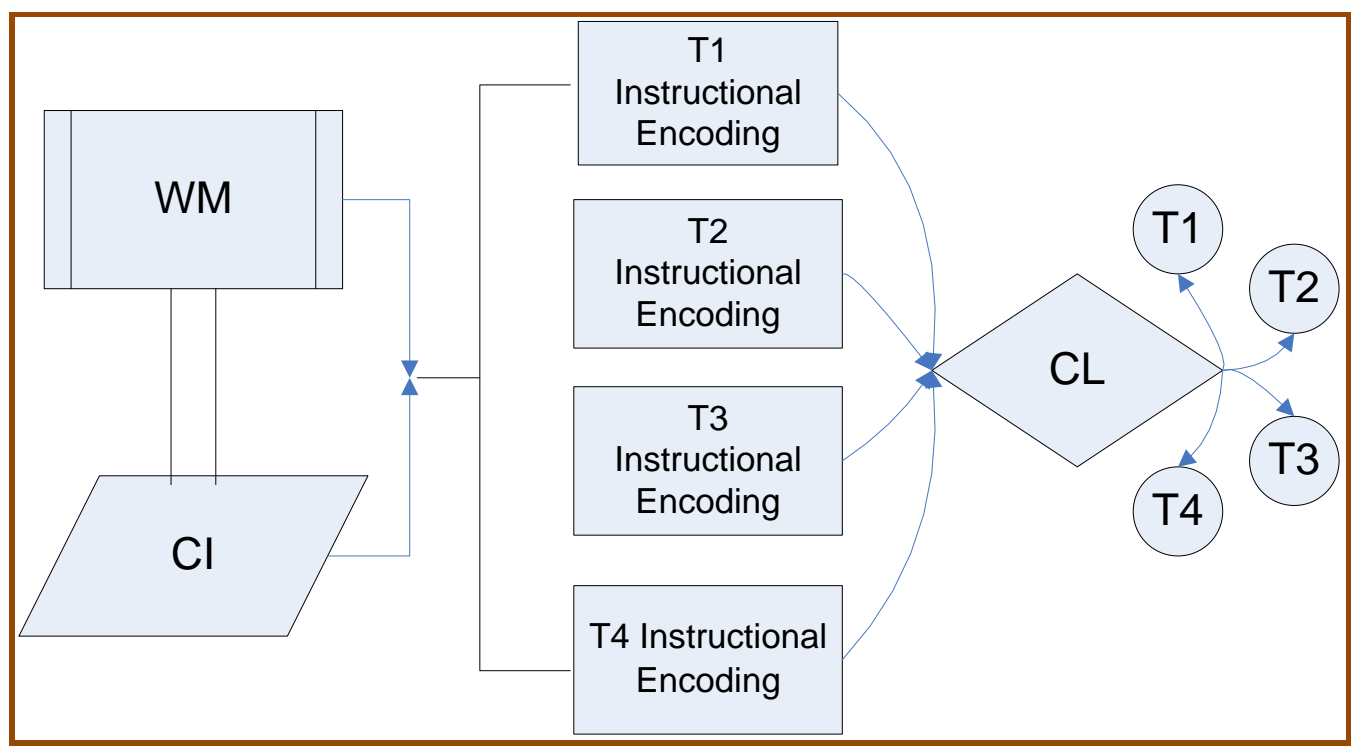

Figure 2: Perceived relationships between WM, inhibition, and cognitive load.

\subsection{Hypotheses}

There were just two hypotheses tested by this initial study: $\mathbf{H}_{1}$ : predicted that student WM function will correlate positively with the students' academic achievement, and $\mathbf{H}_{\mathbf{2}}$ : predicted that significant correlations will occur between the students'

\section{Methods}

The relationship between inhibition and cognitive load was tested by measuring individual student inhibitory ability, and then having students rate how hard they had to work mentally in order to process instructional information under each of the four teachers. It was assumed that a positive, generalised relationship found for inhibition in relation to cognitive load for these students would indicate that more-able inhibitors experience less mental effort in relation to their classroom learning, while less-able inhibitors experience greater mental effort in relation to the same classroom learning. To support the developmental and academic integrity of the student sample in relation to the construct of WM being used in the study, comparisons were also made between relevant WM functions for the students, and their overall academic achievement levels. inhibitory measures and the amount of cognitive load they perceive in relation to learning. The first hypothesis was used to ensure that participating students were processing information at a developmentally appropriate level, and the second to test the inhibition/extrinsic cognitive load assumption.

\subsection{Participants}

Participants in the study included 114 Year-8 students (54\% female, $46 \%$ male), with an average age of 13.83 years $(\mathrm{SD}=0.40)$, and 4 teachers $(1$ female) with an average of 8.5 years teaching experience $(\mathrm{SD}=5.20 \mathrm{yrs}$, range $=2$ to 14 years). Subjects being taught by the teachers included English, Science, History (female), and Math. Using a single year-8 cohort maintained a similar developmental focus across the participant student population, and it is to be noted that the demographics for these students displayed normal population characteristics, including a wide range of SES backgrounds, ethnic diversity, differences in educational backgrounds, and a normally distributed range of prior achievement outcomes. 


\subsection{Ethics approval and sample size}

After obtaining ethics approval for the research, a power analysis was conducted, indicating that the sample size would detect a moderate effect, controlling for type II errors with a statistical power of 0.42 . The theoretical value of the study was considered important, so the investigation was continued.

\subsection{Measures}

A standardised measure of WM capacity, the Operation-Word Span Task [the OSPAN, cf. 14], and a complex measure of selective attentional processing, the Attentional Networks Test [the ANT, cf. 10], were used to measure a range of information processing abilities across the sample of students, including their short-term memory store for word recall, their ability to problem-solve mathematical equations accurately, and their ability to suppress or ignore task-irrelevant or distracting information.

\subsubsection{Cognitive inhibition}

Student inhibitory function was tested using a Flanker task, considered a good overall indicator of inhibitory function in that it measures both the identity and location of task-irrelevant information in relation to attentional focus $[4 ; 11]$. As used here, the Flanker was embedded within the ANT, which is designed to measure differences in attentional control $[5 ; 10]$.
According to Jin Fan (personal communication, April, 2006) attentional control is concerned with how an individual is able to resolve attentional conflict relating to the identity and/or location of information, that is, how well the person is able to retain attentional focus in light of irrelevant or distracting information. The ANT does this by comparing accuracy of identification and response time differences for congruent versus incongruent processing conditions. The measure of this function by the ANT is referred to as "conflict data", and was used here as an indicator of individual differences for inhibition among the participating student population.

\subsubsection{Cognitive load}

To determine cognitive load the students rated each teacher according to how much mental effort they experienced during lessons received from that teacher, that is, according to how "hard" they felt they had to work mentally under instructions from the teacher. They did this by assigning a numerical value on a scale of $1-7$ to the amount of mental effort they experienced during sixteen (16) individual lessons over the course of one academic year, in relation to each of the participating teachers. Individual cognitive load ratings were then averaged for each student, in relation to each of the teachers, to deliver an overall average cognitive load rating as perceived by the students for each teacher.

\section{Analyses}

Table 1 lists the characteristics of the variables viewed as impacting most directly on the research.

Table 1: Overview of variables relating to the research hypotheses

\begin{tabular}{|l|l|l|l|}
\hline Variable & Measurement & Study Mean & SD \\
\hline RECALL (word recall/OSPAN) & Mean word recall & 4.91 & 1.29 \\
\hline ACCURACY (math's operations/OSPAN) & \% (out of 100) & 75.29 & 11.64 \\
\hline CI (Conflict scores on the ANT) & Response Time (in millisecs.) & 184.36 & 87.84 \\
\hline T1_CG.LD (student CL rating for teacher 1) & 7-point scale & 4.12 & .81 \\
\hline T2_CG.LD (student CL rating for teacher 2) & 7-point scale & 4.23 & 1.19 \\
\hline T3_CG.LD (student CL rating for teacher 3) & 7-point scale & 4.04 & .98 \\
\hline T4_CG.LD (student CL rating for teacher 4) & 7-point scale & 3.48 & 1.24 \\
\hline ACHIEVE & \% mark (out of 100) & 71.37 & 10.81 \\
\hline
\end{tabular}

It is to be noted that the variable " $\mathrm{CI}$ " (representing inhibition) is viewed as the independent variable of interest in these analyses. This variable, along with the cognitive load ratings assigned to the four teachers (T1 - T4_CG.LD), outcomes for the specified WM tasks (RECALL and ACCURACY), and the student achievement marks (ACHIEVE), were all normally distributed. Reliability analysis for the student cognitive load ratings returned an overall Cronbach alpha coefficient of .64. The reliability estimate for RECALL X ACCURACY was .68, 
providing support for the WM construct as measured in the study.

\section{Results}

The first hypothesis predicted a significant, positive relationship between WM function and student achievement outcomes. This hypothesis is in keeping with a large body of evidence suggesting that WM function is highly correlated with learning [3]. It is tested here in order to establish that the sample for the current study demonstrated developmentally appropriate levels of WM function.

The WM functions of interest were defined in terms of student scores on the OSPAN relating to representational holding (word recall) and active processing (accuracy in solving math problems), both measures relating to classroom learning and cognitive load theory. Correlations between student achievement outcomes and the WM measures were highly significant, with RECALL and ACHIEVE returning $r\left({ }_{114}\right)=.26, p<.01$, and ACCURACY and ACHIEVE returning $r\left({ }_{114}\right)=.24, p<.02$. A univariate test of between-subjects effects for the relationship between RECALL and ACHIEVE returned $F\left({ }_{108,5}\right)=5.41, p<.04, \eta^{2}=.62$. Betweensubjects effects for ACCURACY and ACHIEVE returned $F(108,5)=4.56, p<.06, \eta^{2}=.58$.

The second hypothesis stated that a significant relationship would be found for student inhibitory function and the cognitive load ratings the students assigned to their learning. In this relationship, the more able inhibitors were expected to assign less cognitive load to their learning, while the less able inhibitors were expected to assign more cognitive load to their learning. Table 2 displays the correlations relating to this hypothesis. Note that this relationship was significant for three out of the four instructional situations experienced by the students, supporting the main hypothesis of the research, which predicted that students more efficient at inhibition also experienced less cognitive load in relation to their learning.

Table 2: Correlations between inhibition ( $\mathrm{Cl}$ ) and cognitive load (cg.ld), by teacher (t1 - t4)

\begin{tabular}{l|rrrr}
\hline \multirow{2}{*}{ CI } & t1_cg.ld & t2_cg.ld & t3_cg.ld & t4_cg.ld \\
\cline { 2 - 5 } & $.228(*)$ & $.198(*)$ & $.186\left(^{*}\right)$ & .136 \\
\hline$n=114$ & & \\
$(*) p<.05,2$-tails
\end{tabular}

In addition, the relationship between CI \& CL seemed to also influence the students' achievement outcomes in relation to the instruction they received from the teachers. Figure 3 shows that in terms of achievement outcomes, a clear interaction occurred between the more able and less able inhibitors, relative to the amount of cognitive load they experienced and in relation to their overall achievement outcomes for the year. Here we see that the less able inhibitors (scoring 169 - 462 on the cognitive inhibition measure) tended to experience both higher levels of cognitive load (CL_Hi) and lower levels of achievement. In contrast to this, the more able inhibitors (scoring 0 - 169 on the cognitive inhibition measure) tended to experience lower levels of cognitive load (CL_Lo) and higher levels of achievement. Of importance is that these two influences seemed to interact just below the mean achievement score of 70, which was the overall achievement mean for the participating student cohort. This supports the division between less and more able inhibitors made in the study, as well as indicating that the experience of cognitive load does have an applied impact on student learning. 


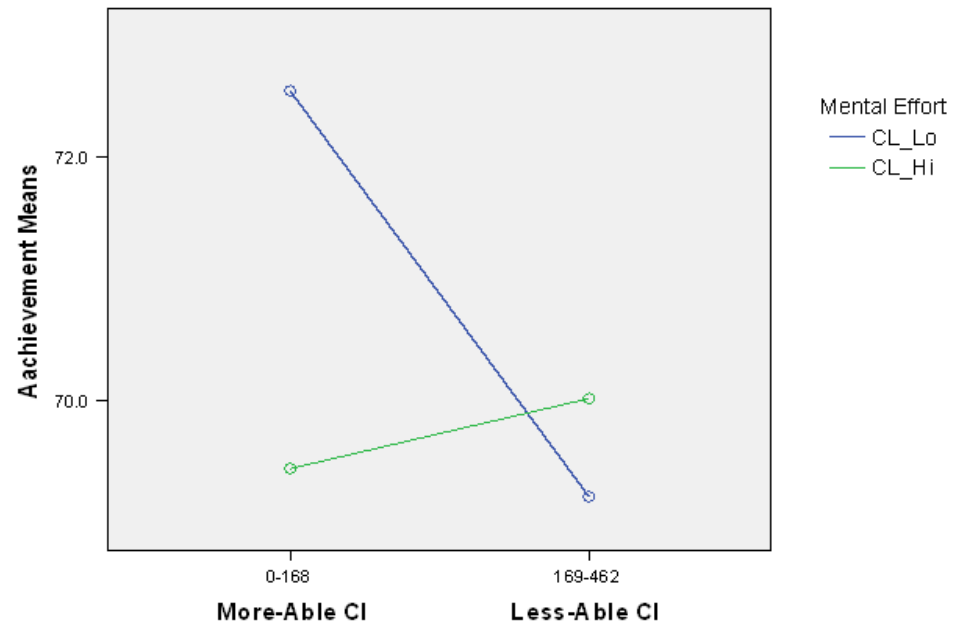

Figure 3: Interaction between cognitive load, cognitive inhibition, and achievement outcomes

\section{Discussion}

The first hypothesis predicted that a significant, positive relationship would exist between WM function and student achievement outcomes, proposing that academic achievement is closely associated with WM function. This hypothesis received strong support, suggesting that instructional designers (including the classroom teacher) need to be aware of the degree to which WM acts as a processing gatekeeper for learning. This finding is in agreement with a body of information that indicates the generalised importance of WM to the learning process, and supports the first hypothesis of the research.

The second hypothesis predicted that a positive relationship would exist between inhibition (CI) and student cognitive load (CL) ratings for the instruction they received. This relationship was predicted on the basis that less-efficient inhibitors were expected to experience greater cognitive load in relation to their learning than the more-efficient inhibitors. Although the overall results obtained for this hypothesis were not unanimous, significant relationships were found for three out of the four participating teachers, indicating that the proposed connection does exist between inhibitory function and mental effort. This is an important finding for the research, and offers general support to the hypothesised relationship of interest.

\section{Study limitations}

Probably the most fundamental limitation with respect to the current study is its intentionally narrow scope, which was designed to examine the effects of instructional encoding factors solely on inhibition and cognitive load. This has meant that other variables (e.g., IQ, SES; etc.) which might help explain these relationships have not been taken into account, and incorporating such variables into future research may prove fruitful. However, the quasiexperimental nature of the current research meant that too much "noise" would have occurred, and possibly obscured authentic findings, unless this more fine-grained focus was adopted at this pilot level of investigation.

\section{Conclusion}

The analyses presented in this study have demonstrated the existence of a generalised relationship between inhibitory processing and mental effort in relation to learning. Most importantly, the findings suggest that variations in cognitive load may be explained in terms of individual differences for inhibitory function, thus signifying that inhibition moderates mental effort in relation to learning. This is an important finding, and suggests that instructional designers need to consider ways to incorporate inhibition-supporting strategies into classroom instruction. 
One obvious application of this finding would be to task-analyse the different phases of complex learning strategies such as Problem-Based Learning (PBL), in order to determine how to control for nonessential processing differentially across the various strategy phases. PBL is a "predictive strategy", that is, a learning strategy that involves students developing hypotheses in order to address a particular problem or theory. In this respect it is important to note that all predictive strategies involve a type of progressive learning. This occurs because the student's efforts to understand and process different predictions about a problem eliminate and refine the predictive elements and information over time. Because of this, there are differences between the amounts of inhibitory activity required to maintain selective attentional focus for early versus later predictive strategy use. This means that the amount of cognitive load can also be expected to vary, according to the amount of representational holding and level of schematic activation involved at different times during the progression.

Figure 4 depicts the relationship between extraneous cognitive load and the use of predictive strategies. This depiction is based on what could be termed the "inhibition $\mathrm{X}$ cognitive load model of attentional processing", as established by the current research. Note that this relationship is driven by the amount of information priming that is associated with the different phases of the strategy, and that the purposes of priming change with the need to address different processing issues that arise as the strategy progresses. This means that different "inhibitory strategies" need to be employed during the early phases of a predictive strategy to those employed in the later phases of the predictive strategy, which is in line with the overall effects of progressive learning across the different phases. The first of these strategies involves the minimisation of misleading instructional influences, and seeks to limit the amount of representational holding and irrelevant activation that occurs during the early phase of prediction. Conversely, the second strategy would then involve the elaboration of facilitating instructional influences, and seek to consolidate and expand the overall learning that has taken place across the strategy.

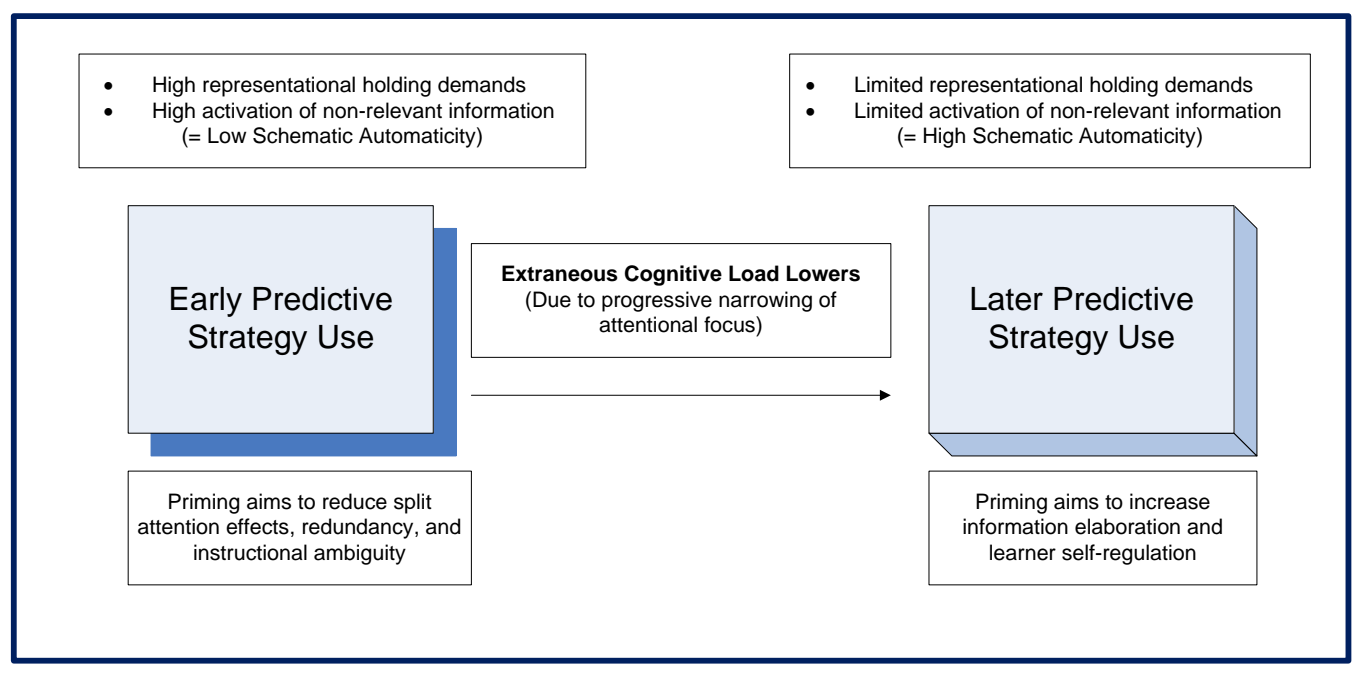

Figure 4: Depiction of predictive strategy use in relation to extraneous cognitive load.

Of additional interest is that this study has identified cognitive inhibition as a likely causal mechanism for explaining the influence of extrinsic cognitive load. This finding is important to Cognitive Load Theory (CLT) because it allows that theory a means of delineating the causal effects of mental effort at a more empirical level than has been prior available, thereby offering CLT a way forward in terms of further empirical testing and extrapolation.
Thus, although the present study has limited scope, it nonetheless offers relevant knowledge and direction for ongoing research in the areas of WM, inhibition, and cognitive load.

\section{Recommendations for future research}

Future investigations of inhibition in relation to CLT should seek to test explicit priming strategies at 
the classroom level, to determine the extent to which priming might be used to affect cognitive load. In this respect it would be valuable to delineate specific instructional encoding elements, and then use coraters to observationally record the degree to which each element is present within the instructional encoding of teachers. This sort of information could be used to explore the relationship between inhibition and cognitive load in a manner that goes beyond the more generalised context of this particular study, and help specify particular elements of instructional encoding that can be linked to the inhibitory function and to the experience of cognitive load. In light of this, further research seeking to expand these findings should focus primarily on particular instructional priming elements, convergent measures of cognitive inhibition, and specific measures of extraneous cognitive load.

\section{References}

[1] Asloun, S., Soury, S., Couillet, J., Giroire, J. M., Joseph, P. A., Mazaux, J. M., \& Azouvi, P. (2008). Interactions between divided attention and workingmemory load in patients with severe traumatic brain injury. Journal of Clinical and Experimental Neuropsychology, 30(4), 481-490.

[2] Ayres, P, \& Paas, F. (2009). Interdisciplinary perspectives inspiring a new generation of cognitive load research. Educational Psychology Review, 21(2) $1-9$.

[3] Conway, A. R. A., Kane, M. J., Bunting, M. F., Hambrick, D. Z., et al. (2005). Working memory span tasks: A methodological review and user's guide. Psychonomic Bulletin \& Review, 12(5), 769787.

[4] Ericksen, B. A., \& Ericksen, C. W. (1974). Effects of noise letters upon the identification of a target letter in a nonsearch task. Perception and Psychophysics, 16: 143-149.

[5] Fossella, J., Posner, M. I., Fan, J., Swanson, J. M., \& Pfaff, D. W. (2002). Attentional phenotypes for the analysis of higher mental function. The Scientific World Journal, 2, 217-223.

[6] Gerjets, P., Scheiter, K., \& Cierniak, G. (2009). The scientific value of Cognitive Load Theory: A research agenda based on the Structuralist view of theories. Educational Psychology Review, 21(2), 4354.
[7] Harnishfeger, K. K., \& Bjorklund, D. F. (1994). A developmental perspective on individual differences in inhibition. Learning and Individual Differences, 3, 331-355.

[8] Mayer, R. E., \& Moreno, R. (2003). Nine ways to reduce cognitive load in multimedia learning. In $\mathrm{R}$. Bruning, C. Horn, \& L. Pytlikzillig (Eds.), Webbased learning: What do we know? Where do we go? (pp. 23-44). Greenwich, CN: Information Age.

[9] Mecklinger, A., Weber, K., Gunter, T. C., \& Engle, R. W. (2003). Dissociable brain mechanisms for inhibitory control: Effects of interference content and working memory capacity. Cognitive Brain Research, 18(1), 26-38.

[10] Posner, M. I., \& Fan, J. (2005). Attention as an organ system. In J. R. Pomerantz and M. C. Crair, (Eds.), Topics in integrative neuroscience: From cells to cognition (pp. 1-34). Cambridge, UK: Cambridge University Press.

[11] Schmidt, P. A., \& Dark, V. J. (1998). Attentional processing of "unattended" flankers: Evidence for a failure of selective attention. Perception \& Psychophysics, 60(2), 227-228.

[12] Schnotz, W., \& Kürschner, C. (2007). A reconsideration of Cognitive Load Theory. Educational Psychology Review, 19(4), 469-508.

[13] Sweller, J. (1994). Cognitive load theory, learning difficulty, and instructional design. Learning and Instruction, 4, $295-312$.

[14] Turner, M. L., \& Engle, R. W. (1989). Is working memory capacity task dependent? Journal of Memory and Language, 28, 127 - 154.

[15] van Merriënboer, J. J. G, \& Sluijsmans, D. M. A. (2009). Toward a synthesis of Cognitive Load Theory, four-component instructional design, and self-directed learning. Educational Psychology Review, 21(5), 55-66. 